\title{
A Chemokine, SDF-1, Reduces the Effectiveness of Multiple Axonal Repellents and Is Required for Normal Axon Pathfinding
}

\author{
Sreekanth H. Chalasani, ${ }^{1}$ Kimberly A. Sabelko, ${ }^{1}$ Mary J. Sunshine, ${ }^{2}$ Dan R. Littman, ${ }^{2}$ and Jonathan A. Raper ${ }^{1}$ \\ ${ }^{1}$ Department of Neuroscience, University of Pennsylvania School of Medicine, Philadelphia, Pennsylvania 19104, and ${ }^{2}$ Howard Hughes Medical Institute, \\ Skirball Institute of Biomolecular Medicine, New York University School of Medicine, New York, New York 10016
}

\begin{abstract}
Altering the concentrations of cyclic nucleotides within nerve cells can dramatically change their responses to axonal guidance cues, but the physiological signals that might induce such alterations are unknown. Here we show that the chemokine stromal cell-derived factor 1 (SDF-1) reduces the repellent activities of slit-2 on cultured retinal ganglion cell axons, of semaphorin 3A on dorsal root ganglion sensory axons, and of semaphorin $3 \mathrm{C}$ on sympathetic axons. This is a modulatory effect because SDF-1 has no detectable attractive or repellent effects on retinal or DRG axons by itself. This modulation is mediated through CXCR4, the receptor of SDF-1, and a pertussis toxin-sensitive G-protein-coupled signaling pathway that induces an elevation of cAMP. The spinal cords of CXCR4 mutant mice contain hyperfasciculated and aberrantly projecting axons. These results suggest that SDF-1 plays an essential role in modulating axonal responsiveness to various known guidance cues through a cyclic nucleotide-dependent signaling pathway.
\end{abstract}

Key words: axon guidance; growth cone; SDF-1; slit-2; semaphorin 3A; semaphorin 3C; repellents; modulation; cAMP; retina; sensory; sympathetic

\section{Introduction}

The growing tip of an axon, the growth cone, senses signals in surrounding tissues that guide it toward its appropriate target. A number of secreted, transmembrane, and phospho-inositide (PI)-linked proteins from the semaphorin, slit, ephrin, and netrin families are thought to act as axonal guidance cues (TessierLavigne and Goodman, 1996). Although the netrins have potent attractant activities, most of the guidance cues characterized thus far appear to act predominantly as repellents. In fact, the presence of so many potent repellents in some regions of the developing nervous system raises the question of how axons are able to extend at all. For example, retinal ganglion cell axons grow immediately adjacent to cells in the optic nerve that produce a powerful chemorepellent, slit-2 (Erskine et al., 2000; Niclou et al., 2000). A plausible conclusion is that either the inherent state of the growing axon or the competing effects of other outside signals sometimes make axons insensitive to repellent guidance cues.

Recent evidence suggests that the modulation of intracellular cyclic nucleotides may play just such a role. Cultured Xenopus spinal neurons are normally repelled by semaphorin $3 \mathrm{~A}$

Received Sept. 17, 2002; revised Nov. 19, 2002; accepted Nov. 20, 2002.

This research was supported by a grant to J.A.R. from the National Institutes of Health (R01-NS26527). We gratefully acknowledge Drs. Klaus Aktories and Gudula Schmidt for providing us with CNF1 and Dr. Bob Doms for providing us with AMD3100. We thank Radhia Ben-Mohamed and Thomas Kreibich for their technical help, Frédéric Baribaud for his help with the chemotaxis assays, and Darlene Ghavimi for making the HIV HXB and JFRL glycoproteins. We thank Drs. Frances Lefcort, Willie Halfter, Judy Meinkoth, Luis Reichardt, and Thomas Jessell for the kind gift of antibodies. We are grateful to Dr. Andrea Webber for help with the mice. We thank Jeffery Masuda-Robbens, Kristin Roovers, and Dr. David Manning for their advice on the signaling pathway. We also thank Greg Bashaw, Thomas Kreibich, and Andrea Webber for their help with this manuscript.

Correspondence should be addressed to Jonathan A. Raper, Professor of Neuroscience, University of Pennsylvania School of Medicine, 1115 Biomedical Research Building II/III, 421 Curie Boulevard, Philadelphia, PA 19104. E-mail: raperj@mail.med.upenn.edu.

Copyright $\odot 2003$ Society for Neuroscience $\quad 0270-6474 / 03 / 231360-12 \$ 15.00 / 0$
(sema3A) or myelin-associated glycoprotein (MAG) (Song et al., 1998). These repellents can be converted to attractants by the addition of pharmacological reagents that elevate the intracellular concentrations of either cAMP or cGMP, respectively. Conversely, netrin can be converted from an attractant into a repellent by lowering the level of cAMP, whereas the attractant neurotrophin-3 can be similarly converted by lowering the level of cGMP (Song et al., 1997). Ligands that trigger changes in intracellular nucleotide levels would be reasonable candidate modulators of axonal responsiveness to guidance cues. For example, the Xenopus data suggest that if a signal induces an elevation of cGMP, it should make axons less responsive to the repellent effects of sema3A than they would be otherwise.

Many seven-transmembrane, G-protein-coupled receptors signal through cyclic nucleotide-dependent pathways (Lameh et al., 1990). This superfamily includes the chemokine receptors. Chemokines are relatively short peptide hormones that were originally defined as chemoattractants for leukocytes but have since been shown to have a much broader spectrum of activities, including promotion of angiogenesis, regulation of $\mathrm{T}$ cell differentiation, and promotion of cell survival (Luster, 1998; Luther and Cyster, 2001). There are roughly 50 chemokines and 20 receptors identified to date. If any of these chemokines affect cyclic nucleotide concentrations inside neurons, the Xenopus data just described suggest that they could reduce or reverse the effects of known repellents such as sema3A. We recently found that SDF-1 has strong survival-promoting effects on cultured embryonic retinal ganglion cells and that this effect is mediated through the G-protein-coupled SDF-1 receptor, CXCR4 (S. H. Chalasani, F. Baribaud, C. M. Coughlan, M. J. Sunshine, V.-M. Lee, R. W. Doms, D. R. Littman, J. A. Raper, unpublished observations). This result shows that embryonic neurons express chemokine 
receptors and respond to chemokines, and as a consequence, raises the possibility that chemokines could modulate axonal guidance cue activities.

We have tested a number of chemokines to determine whether they modulate the effectiveness of axonal chemorepellents. One chemokine, SDF-1, acts through its receptor, CXCR4, to reduce axonal responsiveness to several known repellents. We have also characterized the signaling pathway through which SDF-1 exerts its effects and examined CXCR4 mutant mice to determine whether they have perturbed axon trajectories.

\section{Materials and Methods}

Cultures and growth cone collapse assay. Dorsal root ganglion explants from embryonic day 7 (E7), sympathetic explants from E8, and retinal explants from E6 chicks were cultured in F-12 medium with supplements on glass coverslips coated with laminin (Niclou et al., 2000). For sympathetic cultures, $5 \%$ fetal bovine serum was added to the medium. These cultures were allowed to grow for $18-20 \mathrm{hr}$ before repellents, chemokines, or inhibitors were added. After $30 \mathrm{~min}$, the cultures were fixed and analyzed to determine the percentage of collapsed growth cones. Inhibitors were added to the cultures at the same time as recombinant repellent proteins and SDF-1 $(100 \mathrm{ng} / \mathrm{ml})$. These included a protein kinase A (PKA) inhibitor, PKI-myristoylated (myr) (Calbiochem, LaJolla, CA), at 200 nM; a cAMP antagonist, Rp-adenosine 3', 5' -cyclic monophosphorothioate triethylammonium salt (RpcAMPS) (Sigma, St. Louis, MO), at 20 $\mu \mathrm{M}$; a cGMP antagonist, Rp-guanosine $3^{\prime}, 5^{\prime}$-cyclic monophosphorothioate triethylammonium salt (RpcGMPS) (Sigma), at $20 \mu \mathrm{M}$; a MAP kinase inhibitor, PD98059 (Calbiochem), at $20 \mu \mathrm{M}$; a PI-3 kinase inhibitor, LY294002 (Calbiochem), at $20 \mu \mathrm{M}$; pertussis toxin (Sigma) at 100 $\mathrm{ng} / \mathrm{ml}$; Y-27632 (Tocris Corporation) at $10 \mu \mathrm{M}$, and ML-7 (Biomol, Plymouth Meeting, PA) at 100 nM. An SDF-1 antagonist, AMD3100, at $20 \mu \mathrm{M}$ and $\mathrm{T}$ - and M-trophic glycoproteins, $\mathrm{HxB}$ and JRFL, at 100 $\mathrm{ng} / \mathrm{ml}$ were also used. Cytotoxic necrotizing factor (CNF)- 1 was used at $0.1 \mathrm{nM}$. Agonists such as Sp-adenosine $3^{\prime}, 5^{\prime}$-cyclic monophosphorothioate triethylammonium salt (SpcAMPS) (Sigma) and 8-Bromo-cGMP (8-Br-cGMP) (Sigma) were used at $20 \mu \mathrm{M}$. All of the inhibitors were used at doses that did not interfere with growth cone morphology in the collapse assay.

Cloning and in situ probes. Probes of length 1300 bp, 300 bp, and 2200 bp representing the entire coding sequence of the mouse CXCR4 (PCRs from Incyte clone \#4457694), SDF-1 (\#3675996), and semaphorin 3A were made and used to probe sections from C57BL/6 embryonic mice. Probes were visualized with alkaline phosphatase-conjugated-anti-Dig (Boehringer Mannheim, Mannheim, Germany). The same sections were then reacted with anti-TrkA antibody (a kind gift from Dr. Louis Reichardt, University of California, San Francisco) that was visualized with an anti-rabbit Alexa Fluor 546 (Molecular Probes, Eugene, OR).

cAMP response element-binding protein phosphorylation. Sympathetic chain ganglion from E8 chicks were dissociated and plated on laminincoated glass coverslips in minimal F-12 medium with glucose, glutamine, penicillin, streptomycin, transferrin, and selenium. After $6 \mathrm{hr}$, these cultures were stimulated for 30 min with SDF-1 with and without $20 \mu \mathrm{M}$ AMD3100, $100 \mathrm{ng} / \mathrm{ml}$ pertussis toxin, or $200 \mathrm{~nm}$ PKI. The cultures were then fixed for 20 min with $3.7 \%$ paraformaldehyde and stained with anti-Islet-1 (1:200; 39.4D5, Developmental Studies Hybridoma Bank, Iowa City, IA) and anti-phospho-specific cAMP response elementbinding protein (CREB) (ser-133; a kind gift from Dr. Judy Meinkoth, University of Pennsylvania). These antibodies were then detected by anti-mouse Alexa Fluor 488 and anti-rabbit Alexa Fluor 546 (Molecular Probes).

Collagen matrigels. Three-dimensional cultures of DRG or retinal explants were made using bovine dermis collagen along with F-12 media, supplements, and equal volume of matrigel. 293T cells were transfected with expression plasmids for SDF-1, slit-2, sema3A, or mock ( $\beta$-gal) by calcium phosphate precipitation and transferred into hanging drop cultures for 6-12 hours, and the resulting cell clumps were placed near neural explants. For mixed cultures, individual plasmids were transfected in separate dishes, and then the transfected cells were mixed at equal volumes before they were aggregated together in drop cultures. These cultures received fresh medium with or without SDF-1 as appropriate after $24 \mathrm{hr}$, were fixed after $48 \mathrm{hr}$, and then were stained with antineurofilament (4H6; a gift from Dr. William Halfter, University of Pittsburgh) by a method described in Niclou et al. (2000). Matched cells transfected with SDF- 1 were confirmed to be producing SDF- 1 by Western blot using anti-SDF-1 (R \& D Systems, Minneapolis, MN). The amount of active SDF-1 was estimated with a chemotactic assay using Sup-T1 cells (Hesselgesser et al., 1998) or by measuring its modulatory activity on slit-2 responsiveness in retinal ganglion cells (RGCs). We estimate that sufficient SDF-1 was produced by the average cell clump to make its concentration $500 \mathrm{ng} / \mathrm{ml}$ if distributed uniformly within the matrigel. These cultures were then scored from 1 through 5 as follows: 1 , strong attractant; 3 , no effect; 5 , strong repellent. A parameter-free rank test was then used to calculate statistical significance values ( $p$ values) as shown in Table 1.

Immunohistochemistry. Mouse embryos at day 13.5 were fixed in $4 \%$ paraformaldehyde, cryoprotected in sucrose, and frozen-sectioned. Sections were stained with anti-neurofilament $(2 \mathrm{H} 3, \mathrm{DSHB}, \mathrm{IA})$ at 1:50, which was detected using a goat anti-mouse conjugated to Alexa Fluor 488 (Molecular Probes) at 1:1000. Anti-TrkA (a gift from Dr. Francis Lefcort, University of Montana) was used at 1:1000 and then detected using a goat anti-rabbit conjugated to Alexa Fluor 488 or Alexa Fluor 546 (Molecular Probes) at 1:1000. A guinea pig polyclonal antibody to a motor neuron marker Islet-1/2 was also used at 1:10,000 (a gift from Thomas Jessell, Columbia University).

\section{Results}

\section{SDF-1 reduces the effectiveness of several repellents in growth cone collapse assays}

Growth cones respond to the addition of repellents in the medium by a distinctive morphological change. They withdraw their lamellipodia and filopodia and as a consequence appear "collapsed” (Kapfhammer and Raper, 1987). Slit-2 has a powerful collapsing effect on RGC growth cones, sema3A collapses DRG growth cones, and sema3C collapses sympathetic growth cones (Luo et al., 1993; Koppel et al., 1997; Niclou et al., 2000). The collapse assay has the advantage that repellent activities can be quantified by relating the percentage of growth cones that appear collapsed after the addition of specified amounts of repellent. We used this measure to determine the effectiveness of slit- 2 on RGC growth cones in the presence and absence of various chemokines.

Representative chemokines were chosen in such a way that most receptors in the two largest subclasses of chemokine receptors would be stimulated. These included SDF-1 (CXCL12), GRO $\alpha$ (CXCL1), and IP-10 (CXCL8), which bind to members of the CXC receptor class, as well as MCP-1 (CCL2) and MIP-3 $\alpha$ (CCL20) that bind to members of the CC receptor class (Murphy et al., 2001). All were tested at $100 \mathrm{ng} / \mathrm{ml}$, a concentration that gives strong chemotactic responses for leukocytes (Nanki and Lipsky, 2000). Figure $1 A$ shows a typical RGC growth cone collapse curve as increasing amounts of slit-2 were added to cultures of retinal explants. This dose-response curve was generally unaltered by the addition of most chemokines. In contrast, the addition of SDF-1 shifted the dose-response curve to the right. Approximately eightfold more slit- 2 was required in this experiment to give $50 \%$ collapse when SDF-1 was present in the medium. This factor represents the degree to which SDF-1 reduced slit-2 activity, and it is plotted for each chemokine in Figure $1 B$.

We then determined whether any of these same chemokines reduce the effectiveness of other known repellents. Again, only SDF-1 modulated the collapsing effect of sema3A on DRG growth cones (Fig. 1C), reducing sema3A-induced collapse by fivefold (Fig. 1D). The same pattern emerged with sema3Cinduced collapse of sympathetic growth cones. SDF-1 reduced 

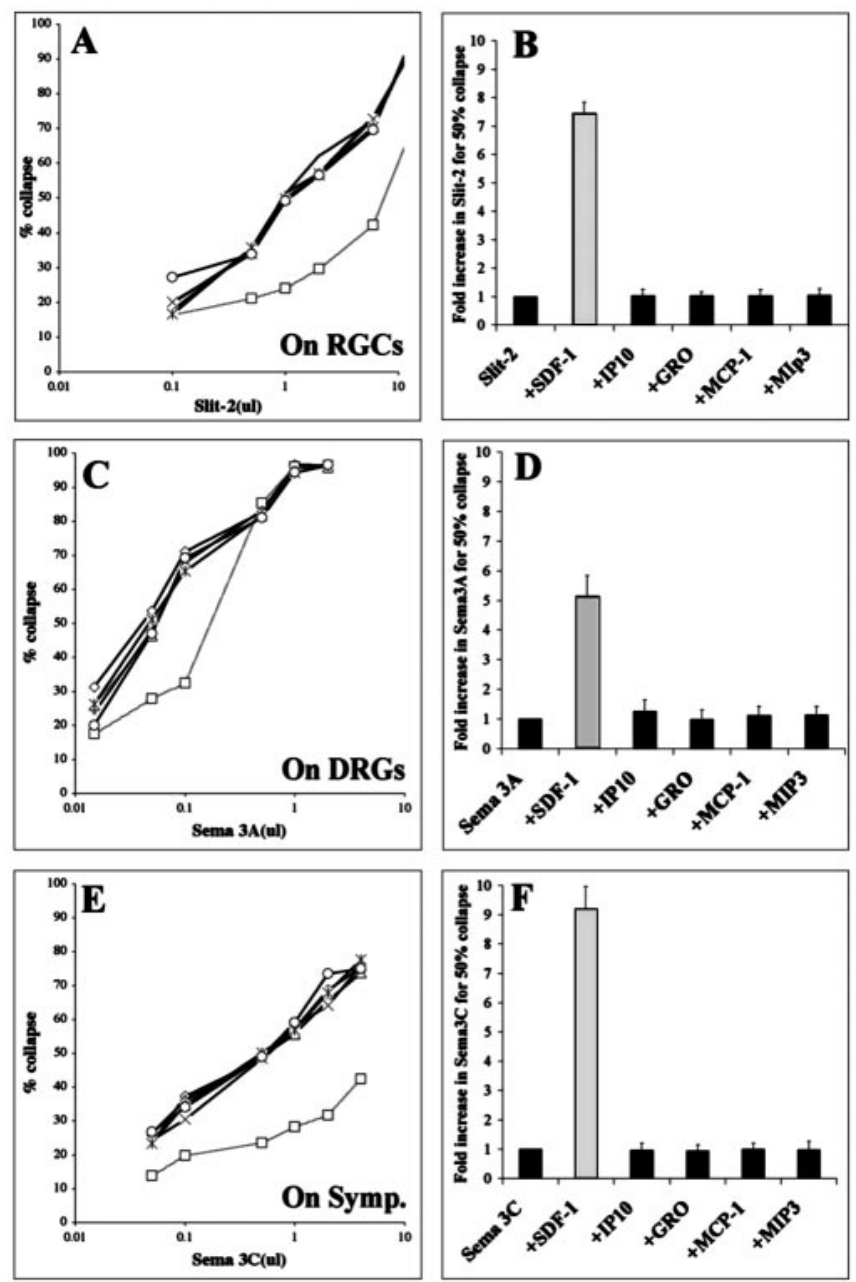

Figure 1. SDF-1 reduces the growth cone collapsing activities of sema-3A, sema-3C, and slit-2. A, The percentages of RGC growth cones collapsed in response to increasing concentrations of supernatant containing recombinant slit-2. Curves representing responses to slit-2 alone (open circles) or in combination with the chemokines IP-10, GRO $\alpha$, MCP-1, or MIP- $3 \alpha$ are indistinguishable, indicating that slit-2 was equally potent alone or in combination with these chemokines. In the presence of SDF-1, higher concentrations of slit- 2 were required to obtain a comparable collapse response (open squares, dotted line), indicating that slit-2 was less effective when combined with SDF-1.B, Fold increase in slit-2 concentration required to achieve $50 \%$ collapse in the presence of selected chemokines. This measure is equivalent to fold reduction in sensitivity to slit-2.C, The percentages of DRG growth cones collapsed in response to increasing concentrations of supernatant containing recombinant sema $3 \mathrm{~A}$ (open circles). In the presence of SDF-1, higher concentrations of sema3A were required to obtain a comparable collapse response (open squares, dotted line). D, Fold increase in sema $3 A$ concentration required to achieve $50 \%$ collapse in the presence of selected chemokines. $E$, The percentages of sympathetic growth cones collapsed in response to increasing concentrations of supernatant containing recombinant sema3C (open circles). In the presence of SDF-1, higher concentrations of sema 3 C were required to obtain a comparable collapse response (open squares, dotted line). $F$, Fold increase in sema3C concentration required to achieve $50 \%$ collapse in the presence of selected chemokines. All chemokines were applied at a concentration of $100 \mathrm{ng} / \mathrm{ml}$.

the effectiveness of sema3C by a factor of approximately ninefold (Fig. 1E, F). These data show that SDF-1 reduces the effectiveness of several repellents in multiple cell types.

\section{SDF-1 is not an attractant for DRG or retinal axons in collagen gel assays}

One explanation for the ability of SDF-1 to reduce growth cone sensitivity to such a wide variety of repellents is that it could act as an attractant. A growth cone that integrates competing repellent
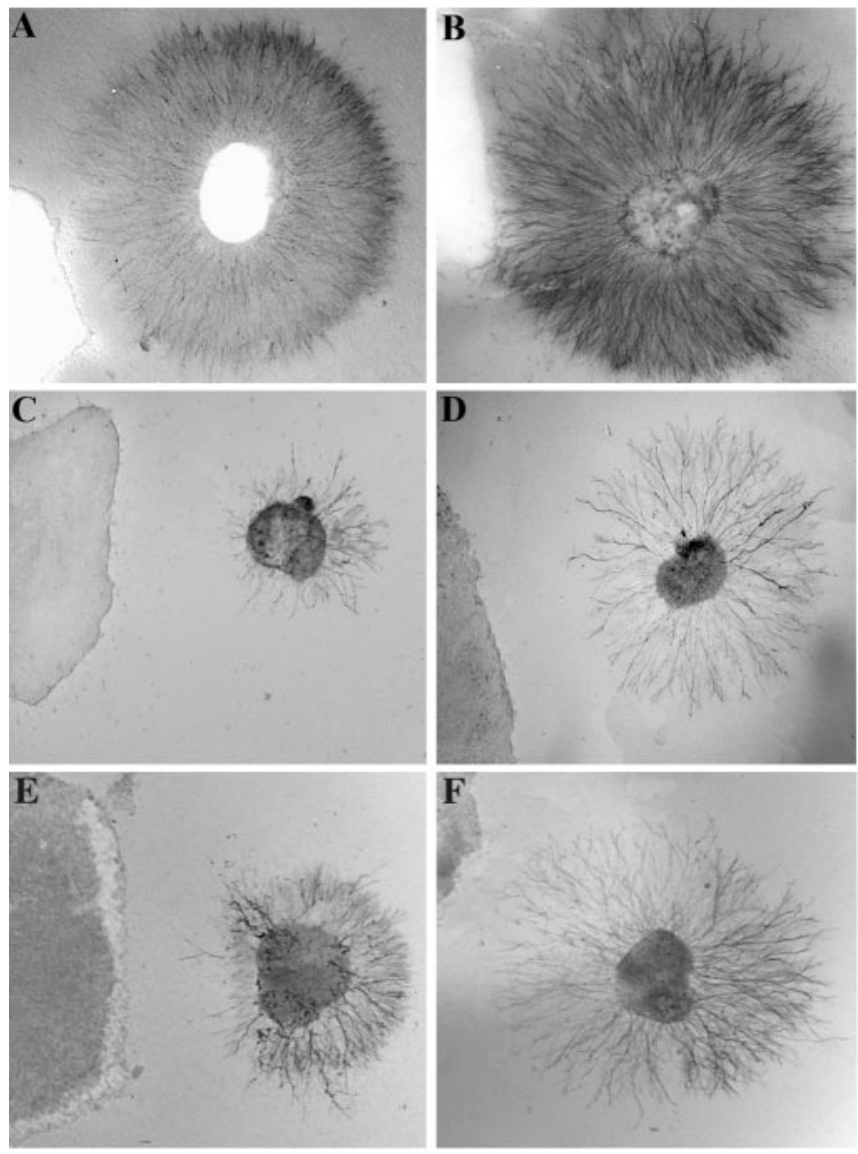

Figure 2. SDF-1 is neither an attractant nor a repellent on its own but reduces repellent activities in a collagen gel assay. E7 DRG explants were embedded in a collagen-matrigel matrix near cell clumps subjected to a mock transfection procedure $(A)$, transfected with SDF-1 $(B)$, transfected with sema $3 A(C)$, transfected with sema $3 A$, and bathed in medium containing 100 $\mathrm{ng} / \mathrm{ml} \mathrm{SDF-1}(D)$. SDF-1 had no repellent or attractant effects of its own but reduced the repellent effect of sema3A. DRGs were placed next to clumps composed of a mixture of sema3A and mock-transfected cells $(E)$, or sema3A and SDF-1 transfected cells ( $F$ ). Mixing SDF-1transfected cells with sema $3 \mathrm{~A}$-transfected cells blocked the repellent effects of sema3A.

and attractant signals might behave as if responding to a weakened repellent. Recent evidence shows that SDF-1 acts as a repellent for rat cerebellar axons (Xiang et al., 2002), an attractant for dentate granule cells (Bagri et al., 2002), and an attractant for embryonic cerebellar neurons (Zhu et al., 2002). To determine whether SDF-1 can act as an attractant for DRG or retinal ganglion cell axons, neural explants were embedded in a collagen gel adjacent to cell clumps secreting recombinant SDF-1. For comparison, explants were also placed near mock-transfected cells. DRG axons grew radially from explants near mock-transfected cells (Fig. $2 A$, Table 1 ). They grew in the same radial pattern near SDF-1-expressing cells (Fig. $2 B$, Table 1). SDF-1 activity was confirmed in supernatants harvested from the same batches of transfected cells used to make cell clumps in the collagen gel cultures. These supernatants had chemotactic activity when tested in a Boydan chamber assay using SupT1 (T-cell line) cells, reacted with anti-SDF-1 antibodies on Western blots of crude supernatant, and reduced the activities of slit-2, sema $3 \mathrm{~A}$, and sema $3 \mathrm{C}$ in collapse assays (data not shown).

Although SDF-1 does not act directly as an attractant or repellent in the collagen gel assay, it can be shown to reduce the activities of known repellents. Overall DRG axon extension was reduced and axons extended farther when growing away from, as 
Table 1. SDF-1 reduces repellent activity of sema $3 A$ and slit-2

\begin{tabular}{|c|c|c|c|c|c|}
\hline Transfected plasmids & Medium & $n$ & $\begin{array}{l}\text { Median } \\
\text { score }\end{array}$ & Like mock? & Like sema? \\
\hline \multicolumn{6}{|c|}{ Dorsal root ganglion cultures } \\
\hline Mock & & 19 & 3 & & № $(p<0.01)$ \\
\hline SDF-1 & & 14 & 3 & $\operatorname{Yes}(p=0.40)$ & № $(p<0.01)$ \\
\hline Sema3A & & 12 & 4 & No $(p<0.01)$ & \\
\hline Sema3A & SDF-1 & 23 & 3 & Yes $(p=0.28)$ & № $(p=0.01)$ \\
\hline Sema $+\beta$-gal & & 18 & 4 & No $(p<0.001)$ & Yes $(p=0.68)$ \\
\hline Sema + SDF-1 & & 10 & 3 & $\operatorname{Yes}(p=0.33)$ & № $(p<0.01)$ \\
\hline \multicolumn{6}{|l|}{ Retinal cultures } \\
\hline Mock & & 28 & 3 & & № $(p<0.01)$ \\
\hline SDF-1 & & 26 & 3 & Yes $(p=0.84)$ & № $(p=0.01)$ \\
\hline Slit-2 & & 21 & 4 & No $(p<0.01)$ & \\
\hline Slit-2 & SDF-1 & 24 & 3 & $\operatorname{Yes}(p=0.77)$ & № $(p<0.01)$ \\
\hline
\end{tabular}

E7 DRG explants were embedded in a collagen-matrigel matrix near cell clumps subjected to a mock transfection procedure (Mock), transfected with SDF-1 (SDF-1), transfected with sema3A (sema3A), a mixture of cells transfected with sema3A and mock-transfected cells (sema3A + mock), or a mixture of cells transfected with sema3A and SDF-1 (sema3A + SDF-1). Each explant was scored as follows: "1" denoting strong attraction, "3" radial outgrowth, and " 5 " strong repulsion as judged by the overall length of the axons extending toward as compared with away from the transfected cell clump. The median score for each condition is given along with a nonparametric rank test estimate of the likelihood that a particular population of scores for any given condition is similar to those for the explants confronted with mock-transfected or sema3A-transfected cells.

compared with growing toward, cells secreting the repellent sema3A (Fig. 2C, Table 1). In contrast, the pattern of axon extension was more symmetrical when recombinant SDF-1 (100 ng/ $\mathrm{ml}$ ) was added to cultures in which DRG explants confronted sema3A-expressing cells (Fig. 2D, Table 1).

To further confirm that SDF-1-mediated signaling reduces axon responsiveness to sema3A and to ensure that active SDF-1 was secreted by transfected cells in our collagen gel assays, cells were separately transfected with SDF-1 and sema3A expression plasmids and then mixed 1:1 before making small cell clumps from them. Control clumps were made from cells separately transfected with $\beta$-galactosidase $(\beta$-gal) and sema3A expression plasmids. Sema3A/ $\beta$-gal control clumps clearly repelled DRG axons (Fig. 2E, Table 1). Although SDF-1 produced in the sema3A/SDF-1 cell clumps did not completely neutralize sema3A activity as judged by the relatively short overall length of sensory axons exiting the neural explants, it was clearly sufficient to reduce the repellent effects of sema3 $\mathrm{A}$ and allow radial axonal outgrowth from explanted DRGs (Fig. $2 F$, Table 1).

SDF-1 was also tested for attractant or repellent activities on retinal ganglion cell axons. Retinal explants cultured near mocktransfected or SDF-1-transfected cell clumps had symmetrical patterns of axon outgrowth (Table 1). Once again, this indicates that SDF-1 alone has no detectable attractant or repellent activity on these axons. Cell clumps expressing slit-2 tended to repel retinal axons. The addition of $100 \mathrm{ng} / \mathrm{ml} \mathrm{SDF-1}$ to the cultures essentially abolished the repellent activity of slit-2. The collagen coculture data indicate that SDF-1 by itself has no detectable attractant or repellent activity on either DRG or retinal axons but support the conclusion from growth cone collapse assays that SDF-1 reduces sema3A and slit- 2 repellent activities.

\section{SDF-1 acts through CXCR4 to reduce repellent activity}

SDF-1 binds and signals through the chemokine receptor CXCR4 in both T cells and neurons (Bleul et al., 1996; Oberlin et al., 1996). We tested specific antagonists of SDF-1 binding to CXCR4 to determine whether they interfere with the ability of SDF-1 to modulate the effectiveness of slit-2. CXCR4 is a coreceptor facilitating HIV-1 entry into T cells (Doranz et al., 1996). The portion of the viral envelope that contacts the chemokine receptor is a glycoprotein, HxB-gp120. This glycoprotein blocks SDF-1 bind- ing to CXCR4 (Staudinger et al., 2001). Another glycoprotein, JRFL-gp120, has been shown to facilitate the infection of macrophages by another HIV strain. JRFL-gp120 does not interfere with SDF-1 binding (Berger et al., 1999). SDF-1 reduced the repellent effects of slit- 2 on retinal axons by a factor of $\sim 12$-fold in the absence of either glycoprotein as measured by the collapse assay (Fig. 3A, compare first and second columns). HxB-gp120, the HIV glycoprotein that interferes with SDF-1 binding to CXCR4, did not affect the repellent effect of slit-2 on RGC axons (Fig. 3A, compare first and third columns), but it abolished the modulatory effect of SDF-1 (Fig. 3A, compare second and fourth columns). A similar result was obtained with AMD3100, a small bicyclam compound that also blocks SDF-1 binding to CXCR4 (Gerlach et al., 2001). AMD3100 by itself did not affect the repellent effect of slit-2 (Fig. 3A, compare first and seventh columns), but adding AMD3100 along with SDF-1 prevented SDF-1-induced modulation of slit-2 repellent activity (Fig. $3 A$, compare second and eighth columns). The HIV glycoprotein that does not interfere with SDF-1 binding to CXCR4, JRFL-gp120, did not block SDF-1 modulation of slit-2 activity (Fig. $3 A$, compare second and sixth columns).

Because CXCR4 is a seven transmembrane G-proteincoupled receptor, pertussis toxin was used to test whether SDF-1 reduces repellent sensitivity via a $G_{i} / G_{o}$ type intermediary (Kaslow et al., 1987). This appears to be the case because pertussis toxin did not affect retinal responsiveness to slit-2 (Fig. $3 A$, first and ninth columns) but blocked SDF-1 modulation of slit- 2 activity (Fig. 3A, compare the second and last columns). Together these results demonstrate that SDF-1 modulates growth cone sensitivity to slit-2 via CXCR4 activation of $G_{i}$.

\section{Reduction of repellent activity by SDF-1 is mediated by elevated cAMP}

Antagonists and agonists of either cAMP or cGMP were used to determine whether SDF-1 modulates slit-2 responsiveness through either of these cyclic nucleotides. The cAMP antagonist RpcAMPS by itself did not affect slit-2-induced collapse (Fig. 3B, compare first and third columns) but did block SDF-1 modulation of slit-2 responsiveness (Fig. 3B, compare second and fifth columns). The cGMP antagonist RpcGMPS affected neither slit-2induced collapse (Fig. 3B, compare first and fourth columns) nor SDF-1 modulation of slit-2 responsiveness (Fig. 3B, compare second and sixth columns). In the absence of SDF-1, the cAMP agonist SpcAMPS mimicked the ability of SDF-1 to reduce responsiveness to slit-2, whereas the cGMP agonist 8BrcGMP did not (Fig. 3B, compare the last two columns). These results indicate that elevating cAMP, but not cGMP, is both necessary and sufficient for SDF-1-induced modulation of slit-2 activity.

Specific inhibitors of selected kinases were tested to determine whether they interfere with SDF-1 modulation of slit-2 activity. The PKA inhibitor, PKI, did not affect responsiveness to slit-2 (Fig. 3C, compare first and third columns) but abolished SDF-1 modulation of slit-2 activity (Fig. 3C, compare second and fourth columns). Inhibitors of MAP kinase (PD98059) or PI-3 kinase (LY294002) did not interfere with SDF-1 activity (Fig. 3C, compare sixth and eighth columns with second column). These results suggest that PKA, known to be a direct target of cAMP action, is required for SDF-1 modulation of slit-2 activity. Both MAP kinase and PI-3 kinase, although downstream of PKA activation, are not required elements in this SDF-1 signaling pathway. In Figure $3 D$ we propose a model for some of the earliest signaling events in SDF-1-mediated modulation of slit-2 responsiveness that is consistent with our data. 
SDF-1 stimulates translocation of cAMP response element-binding protein into the nuclei of sympathetic neurons

Elevated cAMP levels stimulate the phosphorylation of CREB and induce its translocation from the cytoplasm into nuclei (Gonzales and Montminy, 1989; Hagiwara et al., 1993). Translocation can be visualized with antibodies specific for phosphorylated CREB. Sympathetic neurons cultured in fully defined minimal medium have a small amount of phosphorylated CREB distributed diffusely in their cytoplasm (Fig. 4A1-A3). In contrast, neurons exposed to $100 \mathrm{ng} / \mathrm{ml} \mathrm{SDF-1}$ for $30 \mathrm{~min}$ display a dramatic localization of phosphorylated CREB to their nuclei (Fig. 4B1-B3). SDF-1-induced translocation of phosphorylated CREB from the cytoplasm to the nucleus is blocked by AMD3100 (Fig. 4C1-C3), PTX (Fig. 4D1-D3), and the PKA blocker PKI (Fig. 4E1-E3). These findings support the idea that SDF-1 induces an elevation of CAMP through the activation of the CXCR4 receptor and a $\mathrm{G}_{\mathrm{i} / \mathrm{o}}$-mediated signaling pathway.

\section{A role for Rho in SDF-1 modulation of slit-2 activity}

Previous studies have shown that the activation of protein Kinase A in both neuronal (SH-SY) and epithelial-like (SK-EP) cells induces the inactivation of Rho by phosphorylation at Ser-188 (Dong et al., 1998). We hypothesized that the activation of PKA by SDF-1 should have the same effect and that pharmacological agents that activate Rho should block SDF-1 modulation of slit-2 activity. Low doses of CNF1 specifically activate Rho through the deamidation of Gln 63. Higher doses of CNF1 can also activate the other small GTPases Cdc42 and Rac (Flatau et al., 1997; Schmidt et al., 1997). In our experiments, explants were incubated in $0.1 \mathrm{nM}$ CNF1 for $1 \mathrm{hr}$ to avoid activating GTPases other than Rho (Olson et al., 1998). CNF1 by itself did not affect the collapsing activity of slit-2 (Fig. 5A, compare first and third columns). SDF-1 reduced slit-2 activity 12 -fold (Fig. 5A, compare first and second columns). CNF1 partially blocked SDF-1 modulation of slit-2 repellent activity (Fig. 5A, compare second and fourth columns). These results are consistent with the idea that SDF-1 acts through the inactivation of Rho, but these results cannot be viewed as definitive. Only a partial block of SDF-1 activity was obtained and use of higher concentrations of CNF1 would compromise its specificity. However, a role for Rho is further supported by the effects of reagents that block its downstream effectors.

If SDF-1 signaling leads to the inactivation of Rho, then blocking downstream effectors of Rho in the absence of SDF-1 should have the same effect as SDF-1. One important effector of Rho is p160 Rho kinase (ROK) (Bishop and Hall, 2000). An inhibitor of ROK, Y-27632, made retinal axons resistant to slit-2 activity (Fig. $5 B$, compare first and third columns). The addition of SDF-1 had
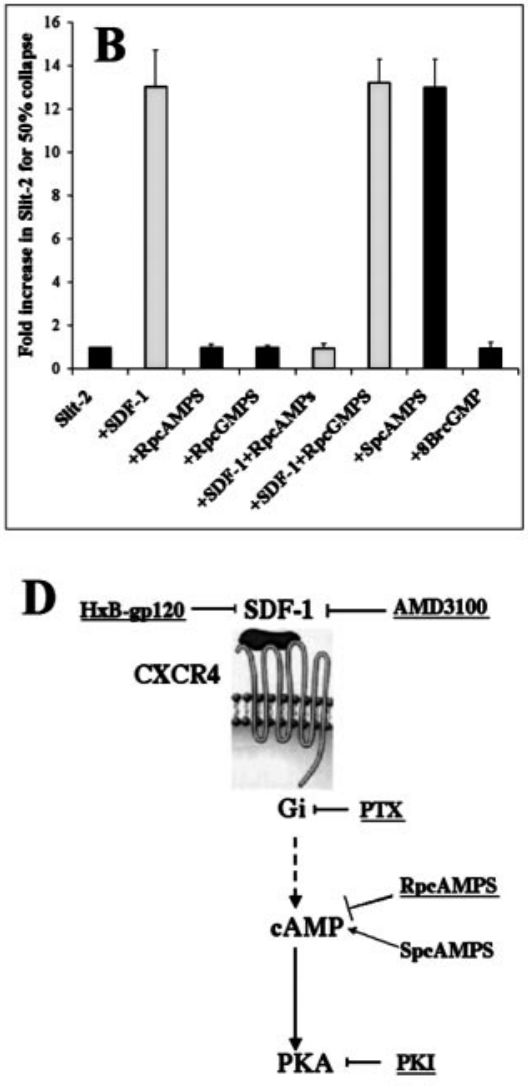

Figure 3. SDF-1 binds CXCR4 and reduces slit-2 repellent activity through a pertussis toxin-sensitive elevation of cAMP and activation plied are indicated with dark bars. See Materials and Methods for concentrations used. A, The HIV-1 coat protein HxB-gp120 and the

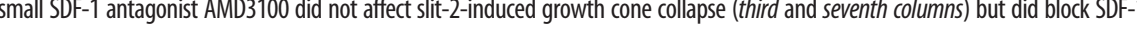
modulation of slit-2 activity ( fourth and eighth columns). A control HIV-1 coat protein that is not an SDF-1 antagonist, JFRL-gp120, did not ( fifth column), nor did it block SDF-1 modulation of slit-2 activity (sixth column). Pertussis toxin cAMPantagonist RpCAMPS blocked SDF-1 modulation of slit-2 activity ( fifth column). The GGMPantagonist RpcGMPS had no effect (sixth column). Neither antagonist affected slit-2-induced growth cone collapse (third and fourth columns). The cAMP agonist SpcAMPS mimicked the ability of SDF-1 to reduce retinal responsiveness to slit-2 (seventh column), whereas the cGMP agonist 8BrcGMP did not (compare second and next to last columns).C, The PKA inhibitor PKI did not affect slit-2 induced growth cone collapse (third column) but blocked SDF-1 slit-2-induced growth cone collapse ( fifth and seventh columns) and also did not block SDF-1 modulation of slit-2 activity (sixth and eighth columns). D, A schematic for a model signaling pathway consistent with the data. Inhibitory interactions are shown with barred lines, whereas activating steps are shown with pointed lines. Broken lines in the signaling pathway indicate multiple intervening steps.

no additional effect over that of Y-27632 on slit-2 responsiveness, consistent with the idea that inhibiting ROK is functionally equivalent to SDF-1 action (Fig. 5B, compare third and fourth columns). One of the direct targets of ROK is myosin light chain kinase (MLCK) (Kimura et al., 1996). An inhibitor of this enzyme, ML-7, also made retinal axons resistant to slit-2 activity (Fig. 5C, compare first and sixth columns). Again, SDF-1 had no additional effect above that of ML-7 alone (Fig. 5C, compare 6th and 10th columns). The reduction of retinal responsiveness to slit-2 that is induced by ML-7 cannot be reversed by PTX, the cAMP antagonist RpcAMPS, or the PKA inhibitor PKI (Fig. 5C, sixth through ninth columns). These results are consistent with the hypothesis that SDF-1 leads to the inactivation of Rho and that the resulting inactivation of first ROK and then MLCK makes RGC growth cones less sensitive to slit- 2 repellent activity (Fig. 5D). Furthermore, they are consistent with MLCKmediated effects on axonal responsiveness being downstream 

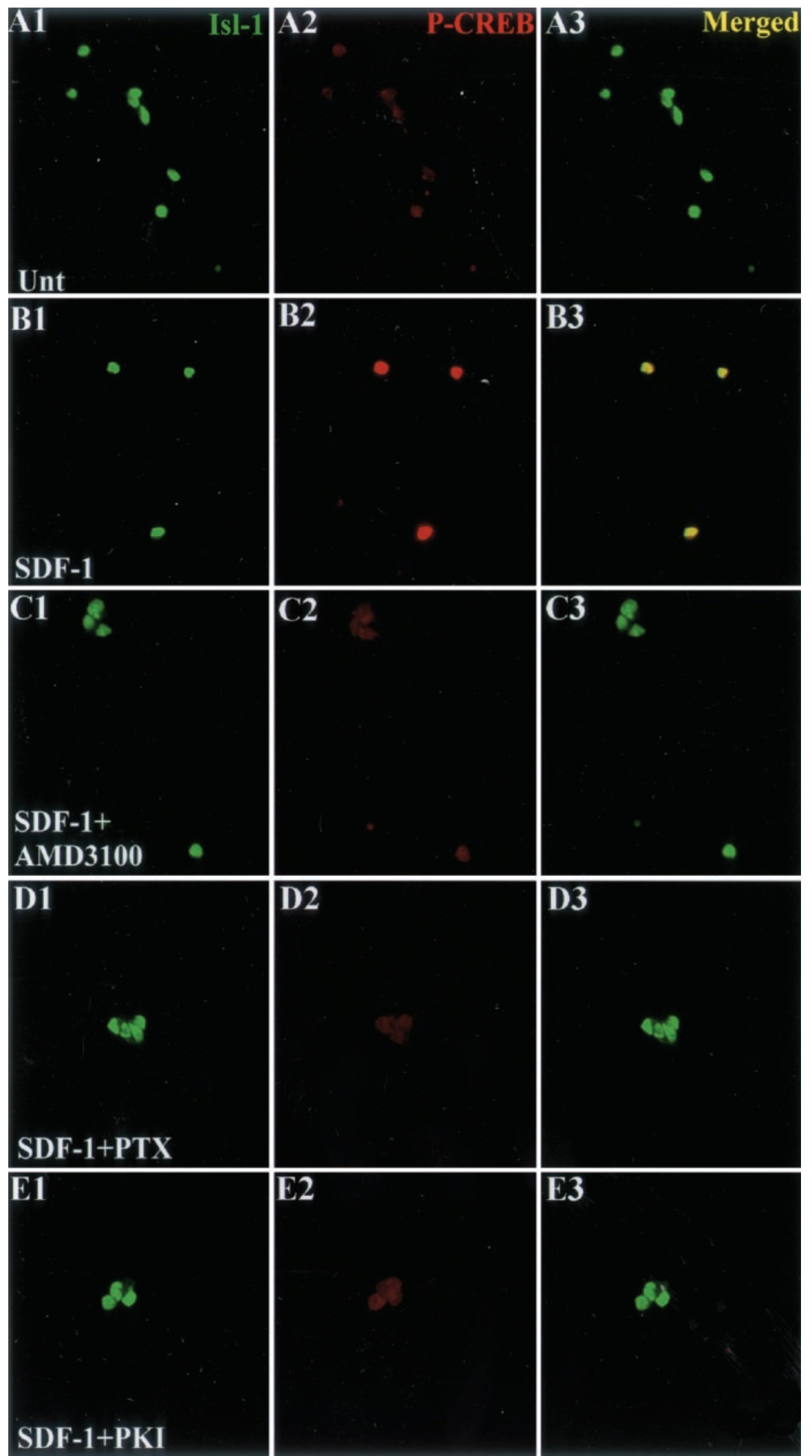

Figure 4. SDF-1 induces translocation of phosphorylated CREB into the nuclei of sympathetic neurons. E8 sympathetic neurons were cultured in defined minimal medium for $6 \mathrm{hr}(A 1-A 3)$ and then exposed to $100 \mathrm{ng} / \mathrm{ml} \mathrm{SDF-1} \mathrm{(B1-B3),} \mathrm{SDF-1} \mathrm{plus} 20 \mu \mathrm{M}$ CXCR4 antagonist AMD3100 (C1-C3), SDF-1 plus $100 \mathrm{ng} / \mathrm{ml}$ pertussis toxin (D1-D3), or SDF-1 plus $200 \mathrm{~nm}$ PKA inhibitor PKI (E1-E3). After $30 \mathrm{~min}$ the cultures were fixed and stained for phosphorylated CREB (red) and Islet-1 (green). SDF-1 induces translocation of CREB into the nuclei of sympathetic neurons, and this translocation is blocked by all three pharmacological agents. from G-protein activation, changes in cAMP concentration, or PKA activation.

SDF-1 modulates slit-2, sema3A, and sema3C activities through the same signaling pathway

Selected inhibitors were used to determine whether SDF-1 reduces axon sensitivity to other repellents through the same signaling pathway. All of the inhibitors that blocked the modulation by SDF- 1 of slit-2 activity in RGCs also blocked the modulation by SDF-1 of sema3A activity on DRGs (Fig. 6A) and sema3C activity on sympathetics (Fig. $6 B$ ). All of the reagents that mimicked the modulation by SDF-1 of slit-2 activity in RGCs also mimicked its modulation of sema3A activity on DRGs (Fig. 6A) and sema3C activity on sympathetics (Fig. 6B). These results suggest that SDF-1 reduces the activity of different repellents in different neuronal types using the same signal transduction pathway.

\section{Embryos lacking CXCR4 contain axons} with abnormal trajectories in the spinal cord

We compared axonal projections within the spinal cords of wild-type and CXCR4 mutant embryos. At E13.5 the axons of Ia muscle spindle afferents originating in the DRGs have entered into the cord and arc ventrally near the dorsal midline (Ozaki and Snider, 1997) (Fig. 7A). Commissural axons have crossed the midline, and ascending and descending projections have generated an extensive rim of axons around the cord. We have detected two unusual phenotypes in CXCR4 mutant animals. Axons traveling in the gray matter are dramatically hyperfasciculated at every cervical and thoracic level in each of the four mutant embryos examined (Fig. $7 B)$. The origin of these axons is unknown, and hence it is difficult to say whether they are misguided. However, this issue can be addressed by examining a subclass of sensory axons. TrkA-expressing cutaneous sensory axons are just beginning to enter the gray matter near the dorsal root entry zone of wild-type E13.5 embryos (Fig. 7C). In contrast, fascicles of these sensory axons invaded the gray matter prematurely in all four of the CXCR4 mutant cords examined. In many instances, these sensory axons first dove deeply into the dorsal cord before looping back outward toward the dorsal rim (Fig. 7D). This behavior was not observed in any of the four matched wild-type littermates examined. These observations show that axon trajectories are abnormal in the spinal cords of CXCR4 mutant mice 
and thus suggest that SDF-1 signaling is required for normal axonal pathfinding.

\section{The relative expression patterns of SDF-1, its receptor CXCR4, and sema3A in the spinal cord}

The ability of SDF-1 to affect retinal and DRG axon responsiveness to repellents suggests that its receptor CXCR4 is expressed in these neurons. As expected, CXCR4 mRNA is expressed in E13.5 mouse DRGS (Fig. 8C3), and in a separate study, we found that it is also expressed in E6 chick and E13.5 mouse retinal ganglion cells (Chalasani, Baribaud, Coughlan, Sunshine, Lee, Doms, Littman, and Raper, unpublished observations). The expression patterns of the SDF-1 receptor CXCR4 were compared with those of slit-2, sema3A, and sema3C in the spinal cord. Slit-2 is expressed at high levels in the floor plate, the roof plate, and in motor neurons (Wang et al., 1999), sema3C is expressed in motor neurons (Zou et al., 2000), and sema3A is expressed in the dermis of the skin epithelium, in motor neurons, and in ventral regions of the cord (Giger et al., 1996; Shepherd et al., 1996) (Fig. 8A1). Even when summed together, the expression patterns of these repellents is confined primarily to the ventral cord. It therefore seems unlikely that SDF-1 modulation of these particular repellents can explain the hyperfasciculation phenotype of axons seen throughout both the dorsal and ventral gray matter of CXCR4 mutant mice. Moreover, the lack of strong CXCR4 expression in cells within the cord suggests that the hyperfasciculated axons seen in the gray matter originate elsewhere, perhaps in the brain or the brainstem.

An interesting relationship between sema3A and SDF-1 expression just outside of the dorsal spinal cord, however, could account for the early entry of TrkA-expressing cutaneous sensory axons. As reported previously, sema3 $\mathrm{A}$ is expressed in the dermis of skin, and this expression is immediately adjacent to the dorsal root entry zone where sensory axons pause before entering the dorsal cord (Fig. 8A1-A3). Interestingly, SDF-1 is expressed in cells ensheathing the cord and the dorsal root nerve (Fig. $8 B 1-B 3$ ). Thus, in normal animals, SDF-1 expressing cells are interposed between sema3Aexpressing dermis and sema3A-sensitive sensory axons. In the absence of SDF-1/CXCR4-mediated signals that reduce sensory axon responsiveness to sema3 $\mathrm{A}$, it seems plausible that sema3 $\mathrm{A}$ might drive sensory cells into the dorsal cord prematurely.

\section{Discussion}

Previous work has shown that raising or lowering levels of cyclic nucleotides can affect a growth cone's responses to axonal guidance molecules (Song et al., 1997, 1998). Thus far, changes in cyclic nucleotide concentrations have been induced using pharmacological agents. The physiological ligands that might induce

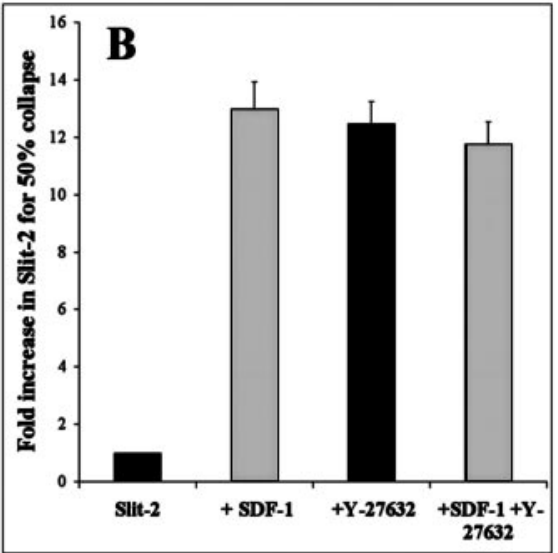

D

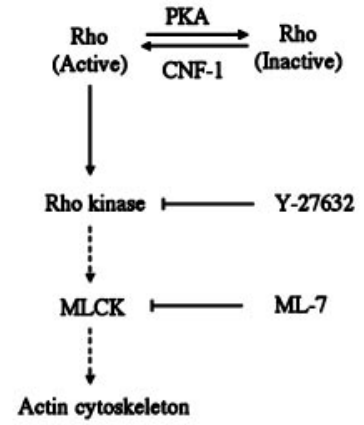

Figure 5. Involvement of Rho and its downstream targets in the SDF-1-activated signaling pathway. Shifts in slit-2 dose-

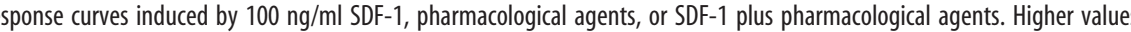
o modulate slit-2 activity ( fourth column). B, The Rho kinase inhibitor Y-27632 mimicked the ability of SDF-1 to modulate slit-2 activity (third column), and SDF-1 had no additional effect ( fourth column). C, The myosin light chain kinase inhibitor ML-7 (n) SDF-1 (seventh, eighth, and ninth columns). D, A schematic of a model signaling pathway consistent with the data (see Results for details). Inhibitory interactions are shown with barred lines, whereas activating steps are shown with pointed lines. Broken lines in the signaling pathway indicate multiple intervening steps.

similar changes have been obscure. Furthermore, because this phenomenon was first described in tissue culture experiments, the relevance of this modulation of responsiveness to axonal guidance cues in vivo was unknown. Here we show that the chemokine SDF-1 reduces the responsiveness of growth cones to several different repellents, that this modulation of responsiveness is mediated by elevated cAMP levels, and that loss of the SDF-1 receptor (CXCR4) leads to perturbations of axonal trajectories in vivo.

Although these studies were in large part inspired by reports that cyclic nucleotide levels modulate and even reverse the sign of growth cone responses to known guidance cues, our results differ from those reported previously in two significant respects. First, we find that neither SDF-1 nor the application of cAMP or cGMP agonists or antagonists at concentrations identical to those used in Xenopus (Song et al., 1997, 1998) convert the repellents that we tested into attractants. Growth cone collapse can still be obtained in cultured chick neurons when a higher dose of repellent is applied. Similarly, in collagen gel assays, repellents are less effective in the presence of SDF-1, but they are not converted to attract- 

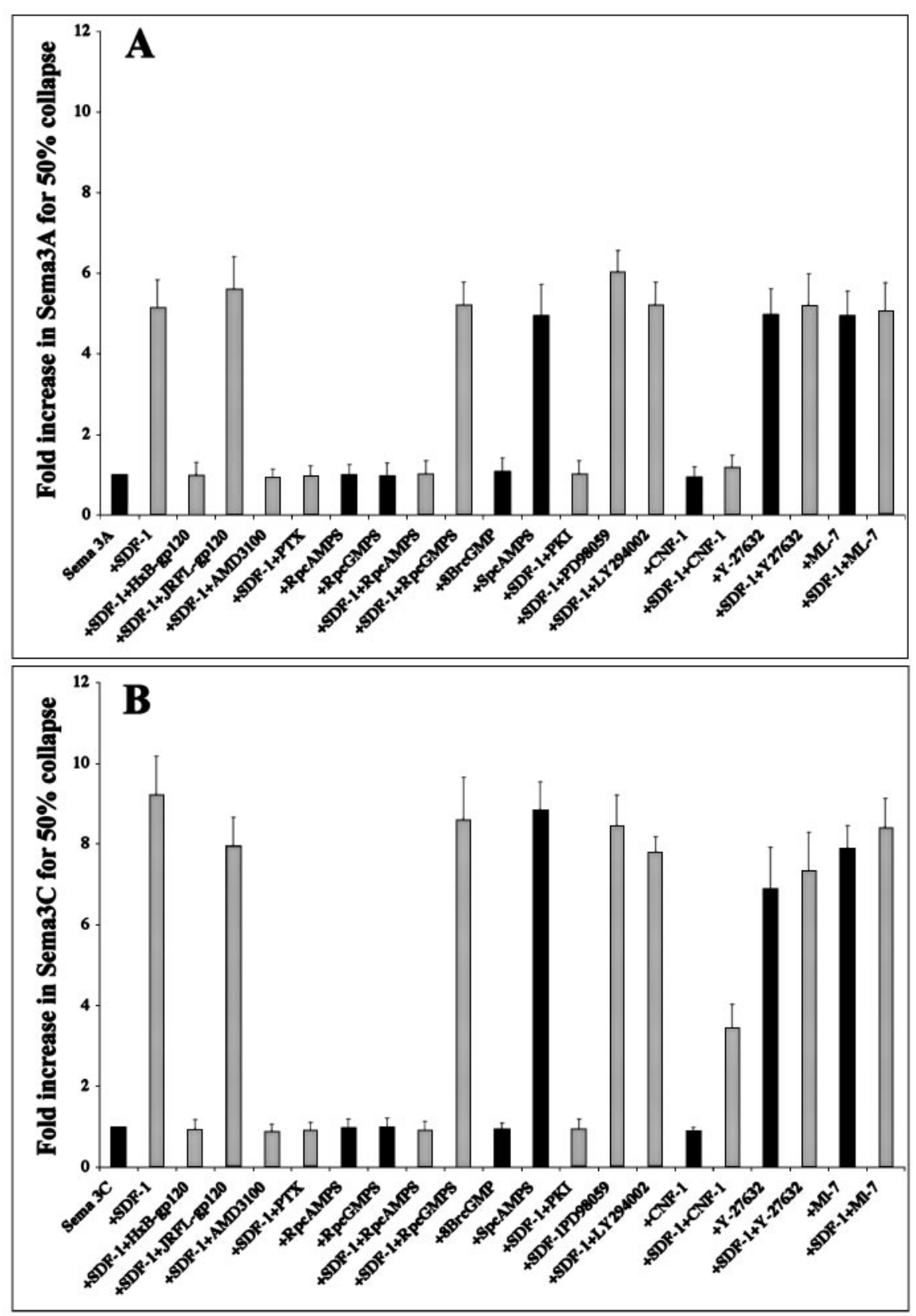

Figure 6. SDF-1 reduces the repellent effects of slit-2, sema3A, and sema3C via the same signaling pathway. Shown are shifts in the dose-response curves for DRG growth cones exposed to sema $3 A(A)$ or sympathetic growth cones exposed to sema $3 C(B)$. Higher values indicate fold increase in repellent concentration required to achieve $50 \%$ collapse. All conditions in which SDF-1 was not applied are indicated with dark bars. Reagents were used at the same concentrations as in the slit- 2 experiments described in Figures 3 and 4. Just as with slit-2 effects on retinal growth cones, the modulatory effects of SDF- 1 on both sema3A and sema3C were blocked by antagonists of SDF-1 binding to CXCR4 (HXB-gp210 and AMD3100), pertussis toxin, an antagonist of CAMP (RpcAMPS), a PKA inhibitor (PKI), and a Rho activator (CNF1), and just as with slit-2 effects on retinal growth cones, the modulatory effects of SDF-1 on both sema3A and sema3 ( were mimicked by a cAMP agonist (SpcAMPS), a Rho kinase inhibitor (Y-27632), and a myosin light chain kinase inhibitor (ML-7).

ants. Second, the specific cyclic nucleotides involved in SDF-1 modulation of repellent activity are not as predicted by the Xenopus results. In Xenopus (Song et al., 1998), elevating cGMP converts sema3A from a repellent to an attractant, but neither cGMP agonists nor antagonists affect chick DRG responses to sema3A. Instead, elevating cAMP in chick neurons reduces the effectiveness of all three repellents tested, including sema3A. One possible reason our results differ from those obtained with Xenopus neurons is that the assays used were different. Repellent activity in the
Xenopus assay was monitored by growth cone turning in response to steep gradients of repellent. Our experiments monitored repellent activity by the differential lengths of axons growing in broad gradients of repellents over long time periods or growth cone collapse induced by a nonlocalized application of repellents over short time periods. The assays that we used have proven to be reliable and in fact were the original method by which these repellents were first identified. Perhaps a more likely explanation for our differing results is that the cellular context in which cyclic nucleotides are altered may determine their effect on guidance cue responsiveness. This is borne out by a report that altering cGMP levels in rodent cortical pyramidal cell dendrites affects their response to sema3A but has no comparable effect on axons of the same cells (Polleux et al., 2000).

Elevating intracellular cAMP through pharmacological intervention has recently been reported to facilitate axonal regeneration in the spinal cords of adult rats (Neumann et al., 2002). Moreover, elevated CAMP levels have been shown to reduce the repellent activity of axons responding to MAG (Qiu et al., 2002). These and our findings are consistent with the idea that elevating cAMP levels in mammalian neurons reduces their responsiveness to repellent guidance cues.

The involvement of Rho in modulation of responsiveness of growth cones to repellents

SDF-1 activates a signaling pathway that elevates cAMP and activates PKA. Activation of PKA inactivates Rho in neuron-like cells (SH-SY) derived from the SK-N-SH cell line by phosphorylating Ser-188 and thereby blocking its interaction with ROK, or in cytotoxic T cells by changing its localization from the membrane to the cytosol (Lang et al., 1996; Dong et al., 1998). Our data are consistent with PKA inducing the functional inactivation of Rho. First, low concentrations of CNF1 toxin are reported to activate Rho (Schmidt et al., 1996; Olson et al., 1998). CNF1 partially blocks the ability of SDF-1 to modulate repellent activities, consistent with the idea that activating Rho works in opposition to SDF-1 activity and PKA activation. Second, inhibition of important targets downstream from Rhomimics the effects of SDF-1. One of the important effectors of Rho is ROK, which inhibits myosin light chain phosphatase,allowing the accumulation of phosphorylated myosin light chains and thereby promoting the formation of acto-myosin filaments (Amano et al., 1996). ROK can also bind myosin light chain directly, but the significance of this interaction is unknown (Bresnick 1999). Con- 

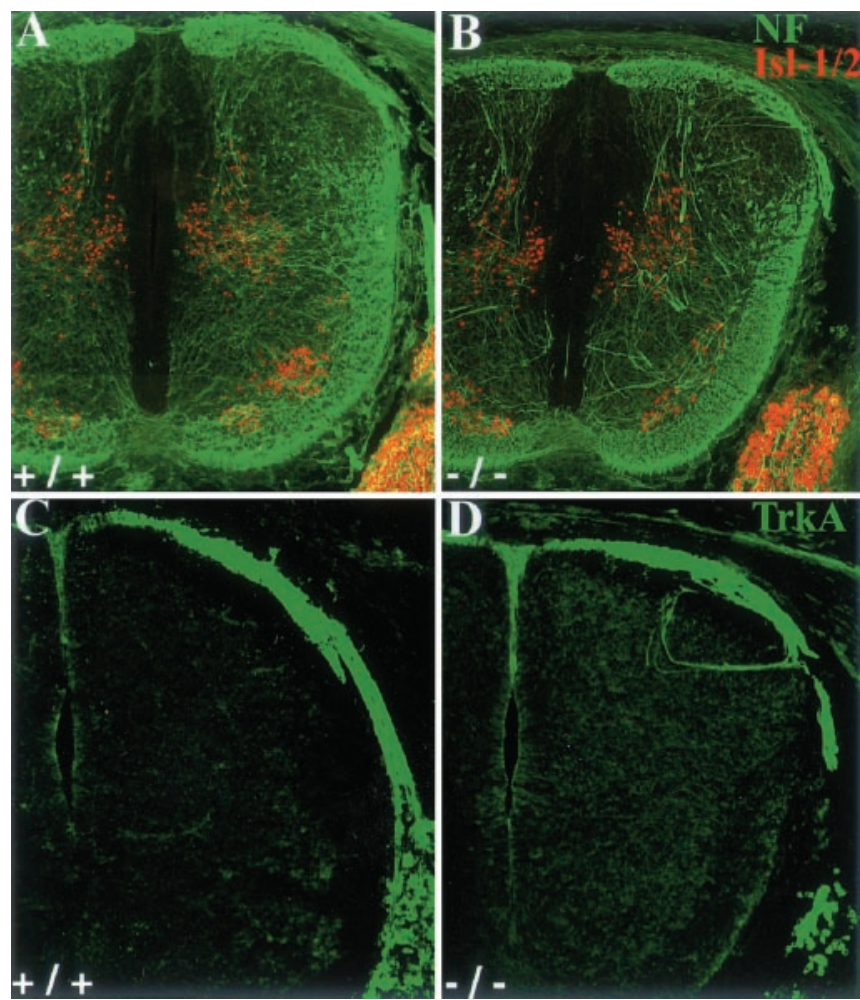

Figure 7. Aberrant axonal trajectories within the spinal cords of CXCR4 mutant mouse embryos. $A, B$, Axons were visualized with anti-neurofilament antibodies (green) and selected neurons with anti-Islet-1 (red) in sections between the second and third cervical levels of spinal cords taken from wild-type $(A)$ and CXCR4 mutant E13.5 $(B)$ littermates. Axons in the gray matter of the cord were hyperfasciculated in all four of the CXCR4 mutant embryos examined as compared with those in wild-type embryos. C, D, Cutaneous sensory axons were visualized with anti-TrkA antibodies in wild-type $(C)$ and XCR4 mutant E13.5 littermates (D). Sections were selected to demonstrate the greatest penetration of Trk A axons in both wild-type and knockouts without trying to match axial level. Fascicles of TrkA-expressing sensory axons entered the dorsal cord prematurely, penetrated deeply, and then turned back toward the dorsal margin in all four of the mutant embryos examined.

versely, Y-27632 blocks the activity of ROK, releasing MLC phosphatase from inhibition, and thereby inactivating MLC (Kimura et al., 1996). The pharmacological reagent ML-7 ultimately has the same MLC inactivating effect by blocking the kinase that activates MLC. Because inhibiting either ROK or MLC kinase has the same effect as exposure to SDF-1, these results are consistent with the hypothesis that SDF-1 prevents their activation by functionally inhibiting Rho.

These results support two models for the ability of SDF-1 to reduce the effectiveness of axonal repellents. In the first model, activation of Rho or its downstream targets is an essential step in the signaling cascade of repellents that is interrupted when SDF-1 blocks Rho activation. In the second model, SDF-1-induced inactivation of Rho acts independently of repellent-induced signals to make the cytoskeleton refractory to repellents.

Current evidence suggests that either model is viable and that they are not mutually exclusive. Recent work in Drosophila implicates Rho in a semaphorin-induced signaling pathway since Plexin-B, a semaphorin receptor, has been shown to bind and activate Rho (Driessens et al., 2001; Hu et al., 2001). Other studies suggest a role for Rho and Rho kinase in ephrin-A5-mediated collapse of retinal growth cones (Wahl et al., 2000). These studies are consistent with a model in which SDF-1-induced signals converge into and block a repellent-induced signaling cascade through the inactivation of Rho.
It may not be essential, however, for SDF-1-induced signals to directly interrupt repellent signaling pathways. Work done mainly in epithelial cells suggests that Rho and its effector proteins play a key role in modulating the actin cytoskeleton (Bishop and Hall, 2000). MLC is thought to induce actomyosin assembly and to promote contraction. It is reasonable to suppose that repellent activity requires the contraction of the actin cytoskeleton of the growth cone. The first repellent, sema3A, was originally described as inducing the morphological conversion of spread and motile growth cones to "collapsed" and paralyzed growth cones (Luo et al., 1993). In principle, SDF-1-induced inactivation of MLC could decrease the contractility of growth cones and thereby make them less responsive to all repellents. One attractive feature of this hypothesis is that all repellents would be made less effective without assuming that they all act through Rho. It might also explain why repellents ultimately remain effective at higher concentrations even in the presence of SDF-1. SDF-1 would reduce growth cone contractility without preventing the profound depolymerization of F-actin triggered by repellents (Fan et al., 1993).

\section{SDF-1 activates bifurcating signaling pathways with separate biological effects}

In a separate study we showed that SDF-1 can act as a neurotrophic factor for embryonic chick and mouse RGCs (Chalasani, Baribaud, Coughlan, Sunshine, Lee, Doms, Littman, and Raper, unpublished observations). The signaling cascade responsible for this biological effect begins similarly to that described here for the modulation of repellent activities. SDF-1 acts through the CXCR4 receptor and via a pertussis toxin-sensitive G-protein to elevate cAMP and activate PKA. However, unlike the repellent modulating activity described here, the survival-enhancing activity of SDF-1 can be blocked with inhibitors of MAP kinase. This suggests that the signaling cascades that modulate repellent responsiveness and the trophic effects diverge below PKA. The repellent modulating pathway is independent of MAP kinase activity and likely acts through Rho to affect the cytoskeleton and repellent signaling pathways, whereas the trophic pathway requires MAP kinase activity and is likely to be directed toward downstream targets involved in cell survival.

\section{Perturbed axonal trajectories in CXCR4 mutant mice}

CXCR4 mutant mice die by E17 and have defects in B-lymphopoiesis, myelopoiesis, cardiac septum formation, and vascular remodeling (Ma et al., 1998; Tachibana et al., 1998; Zou et al., 1998). Consistent with the view that CXCR4 is the only SDF-1 receptor, mice with mutant SDF-1 also die by E17 and have similar developmental defects (Nagasawa et al., 1996; Ma et al., 1998). Two neural defects have been noted in these mice thus far. First, there is an abnormality in cerebellar granule cell migration that could be explained by the demonstrated ability of SDF-1 to act as an attractant for migratory granule cells (Klein et al., 2001; Lu et al., 2001). The attractive effect of SDF-1 produced in the pia is thought to help prevent the premature migration of granule cells into deeper cerebellar layers. Second, we noted a decreased number of retinal ganglion cell neurons in CXCR4 mutant embryos that could be explained by the ability of SDF-1 to support the survival of RGCs in culture (Chalasani, Baribaud, Coughlan, Sunshine, Lee, Doms, Littman, and Raper, unpublished observations). In an initial effort to determine whether axonal trajectories are normal in CXCR4 mutant mice, we surveyed axonal pathways in the embryonic spinal cord after visualizing them with anti-neurofilament antibodies. 

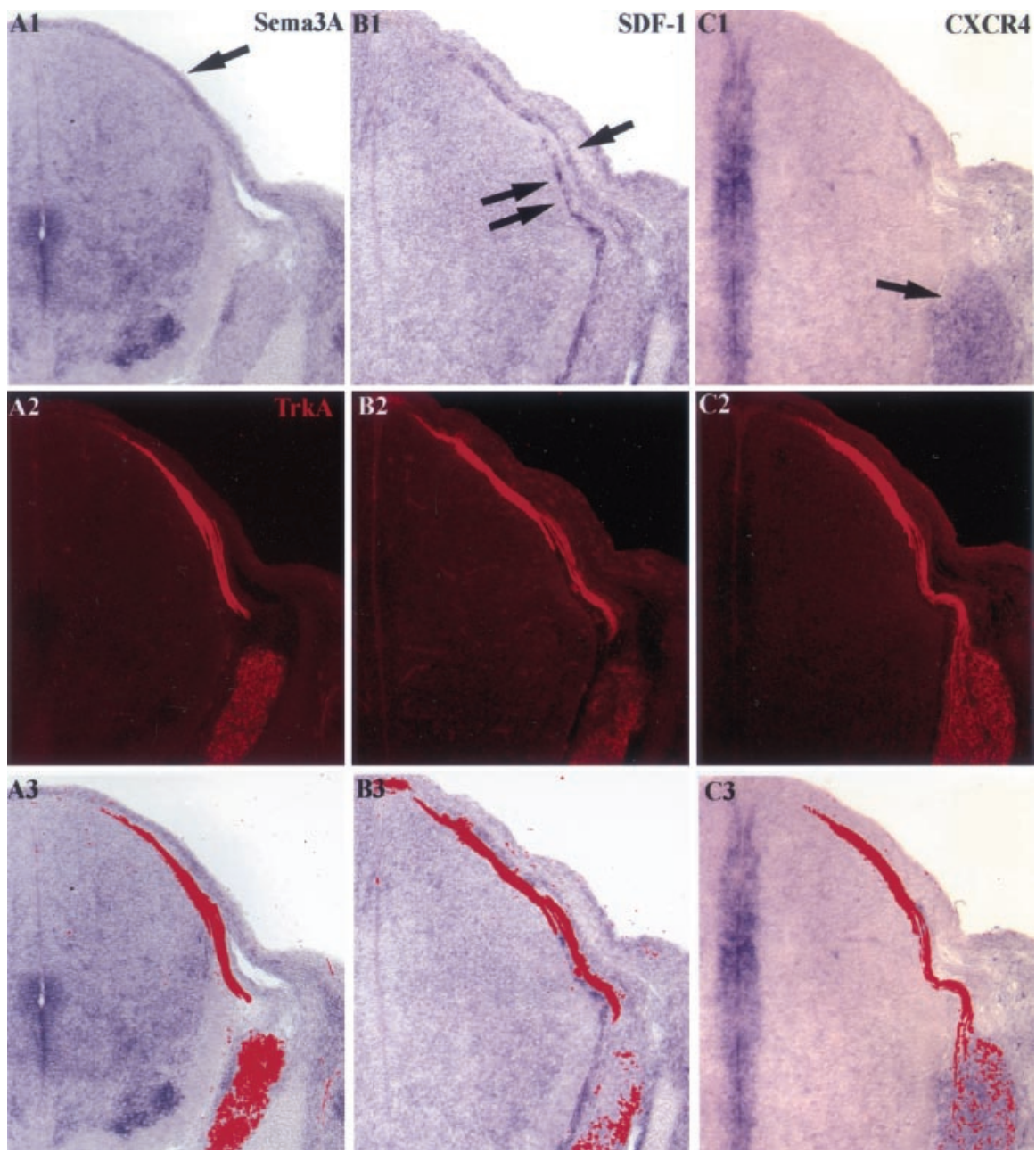

Figure 8. The expression patterns of CXCR4, SDF-1, and sema3C in the mouse E13.5 spinal cord. Sections taken from the spinal cord between $C 2$ and $(4$ were probed with (top row) RNA probes complementary to sema3A (A1), SDF-1 (B1), and CXCR4 (C1). Arrows indicate sema3A expression in the dermis, SDF-1 expression in the sheath surrounding the spinal cord and the dorsal root, and CXCR4 expression in the DRG, respectively. $A 2, B 2, C 2, D R G$ cutaneous sensory axons are visualized with antibodies against TrkA in the same sections probed for mRNA expression. $A 3, B 3, C 3$, The TrkA signal is overlaid on the sema3A, SDF-1, and CXCR4 expression patterns. Note the close proximity of sema3A and SDF-1 expression to the dorsal root entry zone.

A striking phenotype in CXCR4 mutant spinal cords is an apparent hyperfasciculation of axons at all anteroposterior and dorsoventral locations within the cord. A plausible origin for this axonal behavior is the augmentation of repellent activities in the absence of the modulating effects of SDF-1. Axons are more likely to extend on one another when the alternative is to grow through either a nonpermissive or a highly repellent environment (Kapfhammer et al., 1986). Although this interpretation is consistent with the finding that SDF-1 reduces responsiveness to sema3A, sema3C, and slit-2 in vitro, it is unlikely that hyper-responsiveness to any or all of these particular repellents can account for all of the hyperfasciculation that we observe in the CXCR4 mutant cord. At the ages examined, these three repellents are expressed primarily in the ventral and midline regions, whereas hyperfasciculation is observed throughout the cord. Because SDF-1 reduces responsiveness to many, perhaps even all, repellents, augmented activity of other unknown repellents may account for additional hyperfasciculation.

We also examined TrkA-immunoreactive DRG sensory axons that enter the cord through the dorsal root entry zone and grow anteriorly in the dorsal white matter. CXCR4 is expressed in DRGs and SDF-1 modulates the responsiveness of sensory axons to sema3A, consistent with the observation that sensory axon behavior is altered in CXCR4 mutant embryos. Our in vitro results suggest that perturbations in sensory axon trajectories are most likely caused by the loss of SDF-1induced modulation of more traditional guidance cues because SDF-1 has no direct attractant or repellent effects on sensory axons in collagen gel or growth cone collapse assays.

TrkA-expressing sensory processes normally do not begin to extend their first collateral branches into the dorsolateralmost portion of the spinal cord until approximately E14 (Memberg et al., 1995). In contrast, thick fascicles of sensory fibers occasionally enter and penetrate deeply into the dorsal cord of E13.5 CXCR4 mutant embryos. The heavy fasciculation of entering sensory axons is consistent with the hyperfasciculation of neurofilament stained axons already described. The looped appearance of the deeply invading sensory fibers represents a highly unusual trajectory, indicating that sensory axon pathfinding is perturbed. Thus, interfering with SDF-1 signaling in vivo induces premature entry of sensory axons into the CNS as well as hyperfasciculation and misguidance of axons within the cord.

In principle, the early entry of sensory axons in CXCR4 mutant embryos could result from either the augmentation of a repellent outside the cord that drives sensory axons in or augmentation of an attractant inside the cord that draws them in. One possibility is that sema3A repellent activity just outside the cord is potentiated by the loss of SDF-1 signaling, thereby helping to drive sema3A-sensitive sensory axons into the CXCR4 mutant cord prematurely. This interpretation is consistent with the presence of CXCR4 in DRG neurons, the known responsiveness of DRG to sema3A, the expression of sema3A in the dermis of the skin epithelium near the dorsal root, and the expression of SDF-1 just outside the spinal cord near the dorsal root entry zone. We hypothesize that loss of SDF-1/CXCR4-mediated signals makes sema3A produced in the dermis a more effective repellent, thereby driving sensory axons into the cord prematurely. If true, then the loss of sema3 A function would tend to rescue the CXCR4 mutant sensory axon phenotype.

In summary, we have shown that SDF-1 reduces the responsiveness of growth cones to multiple repellents by elevating cAMP levels. Moreover, interfering with SDF-1 signaling induces multiple abnormalities in axon outgrowth in vivo. The wide distribution of SDF-1 in the embryo and its ability to modulate multiple guidance signals suggest that SDF-1 plays an important role in determining the overall effectiveness of repellent cues during development. 


\section{References}

Amano M, Ito M, Kimura K, Fukata Y, Chihara K, Nakano T, Matsuura Y, Kaibuchi K (1996) Phosphorylation and activation of myosin by Rhoassociated kinase (Rho-kinase). J Biol Chem 271:20246-20249.

Bagnard D, Chounlamountri N, Puschel AW, Boltz J (2001) Axonal surface molecules act in combination with semaphorin $3 \mathrm{~A}$ during establishment of corticothalamic projections. Cereb Cortex 11:278-285.

Bagri A, Gurney T, He X, Zou YR, Littman DR, Tessier-Lavigne M, Pleasure SJ (2002) The chemokine SDF1 regulates migration of dentate granule cells. Development 129:4249-4260.

Berger EA, Murphy PM, Farber JM (1999) Chemokine receptors as HIV-1 coreceptors: roles in viral entry, tropism and disease. Annu Rev Immunol 17:657-700.

Bishop AL, Hall A (2000) Rho GTPases and their effector proteins. Biochem J 348:241-255.

Bleul CC, Farzan M, Choe H, Parolin C, Clark-Lewis I, Sodroski J, Springer TA (1996) The lymphocyte chemoattractant SDF-1 is a ligand for LESTR/fusin and blocks HIV-1 entry. Nature 382:829-833.

Bresnick AR (1999) Molecular mechanisms of nonmuscle myosin-II regulation. Curr Opin Cell Biol 11:26-33.

Dong J-M, Leung T, Manser E, Lim L (1998) cAMP induced morphological changes are counteracted by the activated RhoA small GTPase and the Rho kinase ROK $\alpha$. J Biol Chem 273:22554-22562.

Doranz BJ, Rucker J, Yi Y, Smyth RJ, Samson M, Peiper SC, Parmentier M, Collman RG, Doms RW (1996) A dual tropic primary HIV-1 isolate that uses fusin and the chemokine receptors CKR-5, CKR-3 and CKR-2b as fusin cofactors. Cell 85:1149-1158.

Driessens MHE, Hu H, Nobes CD, Self A, Jordens I, Goodman CS, Hall A (2001) Plexin-B semaphorin receptors interact directly with active Rac and regulate actin cytoskeleton by activating Rho. Curr Biol 11:339-344.

Erskine L, Williams SE, Brose K, Kidd T, Rachel RA, Goodman CS, TessierLavigne M, Mason CA (2000) Retinal ganglion cell axon guidance in mouse optic chiasm: expression and function of robos and slits. J Neurosci 20:4975-4982.

Fan J, Mansfield SG, Redmond T, Gordon-Weeks PR, Raper JA (1993) The organization of F-actin and microtubules in growth cones exposed to a brain-derived collapsing factor. J Cell Biol 121:867-878.

Flatau G, Lemichez E, Gauthier M, Chardin P, Paris S, Florentini C, Boquet P (1997) Toxin-induced activation of the G protein p21 Rho by deamidation of glutamine. Nature 387:729-736.

Gerlach LO, Skerlj RT, Bridger GJ, Schwartz TW (2001) Molecular interactions of cyclam and bicyclam non-peptide antagonists with the CXCR4 chemokine receptor. J Biol Chem 276:14153-14160.

Giger RJ, Wolfer DP, De Wit GM, Verhaagen J (1996) Anatomy of rat semaphorin III/collapsin-1 mRNA expression and relationship to developing nerve tracts neuroembryogenesis. J Comp Neurol 18:378-392.

Gonzalez GA, Montminy MR (1989) Cyclic AMP stimulates somatostatin gene transcription by phosphorylation of CREB at serine 133. Cell 59:675-680.

Hagiwara M, Brindle P, Harootunian A, Armstrong R, Rivier J, Vale W, Tsien R, Montminy MR (1993) Coupling of hormonal stimulation and transcription via cyclic AMP-responsive factor CREB is rate limited by nuclear entry of protein kinase A. Mol Cell Biol 13:4852-4859.

Hesselgesser J, Liang M, Hoxie J, Greenberg M, Brass LF, Orsini MJ, Taub D, Horuk R (1998) Identification and characterization of the CXCR4 chemokine receptor in human $\mathrm{T}$ cell lines: ligand binding, biological activity, and HIV-1 infectivity. J Immunol 160:877-883.

$\mathrm{Hu}$ H, Marton TF, Goodman CS (2001) Plexin B mediates axon guidance in Drosophila by simultaneously inhibiting active Rac and enhancing RhoA signaling. Neuron 32:39-51.

Kapfhammer J, Grunewald BG, Raper JA (1986) The selective inhibition of growth cone advancement by specific neurites in culture. J Neurosci 6:2527-2534.

Kapfhammer JP, Raper JA (1987) Interactions between growth cones and neurites growing from different neural tissues in culture. J Neurosci 5:1595-1600

Kaslow HR, Lim LK, Moss J, Lesikar DD (1987) Structure-activity of the activation of pertussis toxin. Biochemistry 26:123-127.

Kimura K, Ito M, Amano M, Chihara K, Fukata Y, Nakafuku M, Yamamori B, Feng J, Nakano T, Okawa K, Iwamatsu A, Kaibuchi K (1996) Regulation of myosin phosphatase by Rho and Rho-associated kinase (Rho-kinase). Science 273:245-248.
Klein RS, Rubin JB, Gibson HD, DeHaan EN, Alvarez-Hernandez X, Segal RA, Luster AD (2001) SDF-1 alpha induces chemotaxis and enhances Sonic hedgehog-induced proliferation of cerebellar granule cells. Development 128:1971-1981.

Koppel A, Feiner L, Kobayashi H, and Raper JA (1997) A 70 amino acid region within the semaphorin domain activates specific cellular response of semaphorin family members. Neuron 19:531-537.

Lameh J, Cone RI, Maeda S, Philip M, Corbani M, Nadasdi L, Ramachandran J, Smith GM, Sadee W (1990) Structure and function of G-protein coupled receptors. Pharmacol Res 12:1213-1221.

Lang P, Gesbert F, Delespine-Carmagnat M, Stancou R, Pouchelet M, Bertoglio J (1996) Protein kinase A phosphorylation of RhoA mediates the morphological and functional effects of cyclic AMP in cytotoxic lymphocytes. EMBO J 15:510-519.

Lu Q, Sun EE, Klein RS, Flanagan JG (2001) Ephrin-B reverse signaling is mediated by a novel PDZ-RGS protein and selectively inhibits G proteincoupled chemoattraction. Cell 105:69-79.

Luo Y, Rabile D, Raper JA (1993) Collapsin: a protein in brain that induces the collapse and paralysis of neuronal growth cones. Cell 75:217-227.

Luster AD (1998) Mechanisms of disease: chemokines-chemotactic cytokines that mediate inflammation. N Engl J Med 338:436-445.

Luther SA, Cyster JG (2001) Chemokines as regulators of T cell differentiation. Nat Immunol 2:102-107.

Ma Q, Jones D, Borghesani PR, Segal RA, Nagasawa T, Kishimoto T, Bronson RT, Springer TA (1998) Impaired B-lymphopoiesis, myelopoiesis and derailed cerebellar neuron migration in CXCR4 and SDF-1 deficient mice. Proc Natl Acad Sci USA 95:9448-9453.

Memberg SP, Hall AK (1995) Proliferation, differentiation and survival of rat sensory neuron precursors in vitro require specific trophic factors. Mol Cell Neurosci 6:323-335.

Murphy PM, Baggiolini M, Charo IF, Hébert CA, Horuk R, Matsushima K, Miller LH, Oppenheim JJ, Power CA (2001) International union of Pharmacology. XXII. Nomenclature for chemokine receptors. Pharmacol Rev 52:145-176.

Nagasawa T, Hirota S, Tachibana K, Takakura N, Nishikawa S, Kitamura Y, Yoshida N, Kikutani H, Kishimoto T (1996) Defects in B-cell lymphopoiesis and bone-marrow myelopoiesis in mice lacking the $\mathrm{CXC}$ chemokine PBSF/SDF-1. Nature 382:635-638.

Nanki T, Lipsky PE (2000) Cutting edge: stromal cell-derived factor-1 is a co-stimulator for CD4 ${ }^{+} \mathrm{T}$ cell activation. J Immunol 164:5010-5014.

Neumann S, Bradke F, Tessier-Lavigne M, Basbaum AI (2002) Regeneration of sensory axons within the injured spinal cord induced by intraganglionic cAMP elevation. Neuron 34:885-893.

Niclou SP, Jia L, Raper JA (2000) Slit2 is a repellent for retinal ganglion cell axons. J Neurosci 20:4962-4974.

Oberlin E, Amara A, Bachelerie F, Bessia C, Virelizier JL, Arenzana-Seisdedos F, Schwartz O, Heard JM, Clark-Lewis I, Legler DF, Loetscher M, Baggiolini M, Moser B (1996) The CXC chemokine SDF-1 is a ligand for LESTR/fusin and prevents infection by T-cell-line-adapted HIV-1. Nature 382:833-835.

Olson MF, Paterson HF, Marshall CJ (1998) Signals from Ras and Rho GTPases interact to regulate expression of p21 Waf1/Cip1 . Nature 394:295-299.

Ozaki S, Snider WD (1997) Initial trajectories of sensory axons towards laminar targets in the developing mouse spinal cord. J Comp Neurol 380:215-229.

Polleux F, Morrow T, Ghosh A (2000) Semaphorin 3A is a chemoattractant for cortical apical dendrites. Nature 404:567-573.

Puschel AW, Adams RH, Betz H (1996) The sensory innervation of the mouse spinal cord may be patterned by differential expression of and different responsiveness to semaphorins. Mol Cell Neurosci 7:419-431.

Qiu J, Cai D, Dai H, McAtee M, Hoffman PN, Bregman BS, Filbin MT (2002) Spinal axon regeneration induced by elevation of cyclic AMP. Neuron 34:895-903.

Schmidt G, Sehr P, Wilm M, Selze J, Mann M, Aktories K (1996) Gln 63 of Rho is deamidated by Escherichia coli cytotoxic necrotizing factor-1. Nature 387:725-729.

Shepherd I, Luo Y, Raper JA, Chang S (1996) The distribution of collapsin-1 mRNA in the developing chick nervous system. Dev Biol 173:185-199.

Song H-J, Ming G, Poo M-M (1997) cAMP-induced switching in turning direction of nerve growth cones. Nature 388:275-279.

Song H-J, Ming G, He Z, Lehmann M, McKerracher L, Tessier-Lavigne M, 
Poo M-M (1998) Conversion of neuronal growth cone responses from repulsion to attraction by cyclic nucleotides. Science 281:1515-1518.

Staudinger R, Wang X, Brandés JC (2001) HIV-1 envelope is a neutral antagonist to CXCR4 in T cells. Biochem Biophys Res Commun 280:1003-1007.

Tachibana K, Hirota S, Iizasa H, Yoshida H, Kawabata K, Kataoka Y, Kitamura Y, Matsushima K, Yohida N, Nishikawa S, Kishimoto T, Nagasawa $\mathrm{T}$ (1998) The chemokine receptor CXCR4 is essential for vascularization of the gastrointestinal tract. Nature 393:591-594.

Tessier-Lavigne M, Goodman CS (1996) The molecular biology of axon guidance. Science 274:1123-1133.

Wahl S, Barth H, Ciossek T, Aktories K, Mueller BK (2000) Ephrin-A5 induces collapse of growth cones by activating Rho and Rho kinase. J Cell Biol 149:263-270.

Wang KH, Brose K, Arnott D, Kidd T, Goodman CS, Henzel W, Tessier-Lavigne
M (1999) Biochemical purification of a mammalian slit protein as a positive regulator of sensory axon elongation and branching. Cell 96:771-784.

Xiang Y, Li Y, Zhang Z, Cui K, Wang S, Yuan X-B, Wu C-P, Poo M-M, Duan $S$ (2002) Nerve growth cone guidance mediated by $\mathrm{G}$ protein-coupled receptors. Nat Neurosci 5:843-848.

Zhu Y, Yu T, Zhang XC, Nagasawa T, Wu JY, Rao Y (2002) Role of the chemokine SDF-1 as the meningeal attractant for embryonic cerebellar neurons. Nat Neurosci 5:719-720.

Zou Y, Stoeckli E, Chen H, Tessier-Lavigne M (2000) Squeezing axons out of the gray matter: a role for slit and semaphorin proteins from midline and ventral spinal cord. Cell 102:363-375.

Zou Y-R, Kottmann AH, Kuroda M, Taniuchi I, Littman DR (1998) Function of the chemokine receptor CXCR4 in haematopoiesis and in cerebellar development. Nature 393:595-599. 